Clio. Femmes, Genre, Histoire

1 | 1995

Résistances et Libérations France 1940-1945

\title{
L'oubli des femmes dans l'historiographie de la Résistance
}

Rita THALMANN

\section{(2) OpenEdition}

1 Journals

Édition électronique

URL : https://journals.openedition.org/clio/513

DOI : $10.4000 /$ clio. 513

ISSN : 1777-5299

Éditeur

Belin

Édition imprimée

Date de publication : 1 avril 1995

ISSN : 1252-7017

Référence électronique

Rita THALMANN, «L'oubli des femmes dans l'historiographie de la Résistance », Clio. Femmes, Genre, Histoire [En ligne], 1 | 1995, mis en ligne le 01 janvier 2005, consulté le 23 avril 2022. URL : http:// journals.openedition.org/clio/513; DOI : https://doi.org/10.4000/clio.513

Ce document a été généré automatiquement le 23 avril 2022

Tous droits réservés 


\title{
L'oubli des femmes dans l'historiographie de la Résistance
}

\author{
Rita THALMANN
}

1 Cinquante ans après la libération de la France, alors que disparaissent progressivement les derniers témoins, le rôle des femmes dans la Résistance, ce que Catherine Varlin (Judith Heytin), lycéenne engagée dès 1941 , appelle " une ville engloutie $»^{1}$, reste encore à écrire. Esquisse de recherches effectuées pour deux colloques $^{2}$ et les catalogues des expositions, l'une temporaire au Musée de la Résistance et de la Déportation de Besançon, l'autre permanente au Centre de la Résistance et de la Déportation de Lyon, cette contribution voudrait rendre une visibilité à cette histoire longtemps occultée. Elle est centrée sur trois questions : qui étaient ces femmes et quelles étaient leurs motivations? Quel fut leur rôle dans la Résistance ? Pourquoi ne tiennent-elles pratiquement aucune place dans l'historiographie, voire, à de rares exceptions près, dans la mémoire officielle de la France?

Qui étaient ces femmes et quelles étaient leurs motivations?

3 Sans minimiser le rôle des hommes, force est de constater que la prise de conscience, qu'impliquait l'engagement dans le combat contre l'occupant et la collaboration de l'État vichyste, était plus difficile pour les femmes, compte tenu de leur statut dans la société française de l'époque, pour au moins trois raisons. D'abord, parce que leur statut juridique, politique et social en faisait des marginales de la vie publique. Ensuite, parce que la majorité d'entre elles ne disposaient pas comme les hommes - sauf celles qui appartenaient à un mouvement de jeunesse - de réseaux susceptibles de les préparer à l'aguerrissement physique que donne la pratique des sports, à fortiori le service militaire. Enfin, parce que l'image traditionnelle de «la» femme, épouse et mère, véhiculée par l'éducation et les médias, les liait davantage à la famille, développant, par voie de conséquence, un plus grand besoin de sécurité et un sens souvent profond de culpabilité chez celles que leur action de résistance obligeait à s'éloigner des leurs pour les protéger de la répression ou privait, comme j'ai pu le constater à travers certains témoignages, d'être ou de devenir mères. 
Ces trois facteurs expliquent sans doute pourquoi ce sont essentiellement des jeunes femmes non mariées, souvent même des lycéennes de 16 à 18 ans, qui constituèrent le fer de lance de la Résistance féminine. Pourquoi, aussi, on trouve une si forte proportion de femmes d'origine étrangère, en particulier d'immigrées d'Europe de l'Est, issues d'un milieu que son itinéraire d'exil avait, par la force des choses, préparé à s'adapter aux dures conditions matérielles et morales du déracinement, à prendre davantage conscience des réalités politiques et à affronter les situations les plus inattendues. À cette immigration provoquée au tournant du siècle par la répression tsariste puis par la guerre civile, s'était ajoutée celle du IIIe Reich, c'est-à-dire de l'Allemagne hitlérienne et des pays déjà occupés ou annexés entre 1938 et 1940. De même que les réfugiés de l'Italie fasciste et de l'Espagne franquiste, qui avaient tous et toutes subi la répression, y compris ceux et celles arrivés dans la France de la deuxième moitié des années trente, hormis la brève embellie du Front populaire ${ }^{3}$. Ce qui ne les empêchait pas de faire la différence entre la tradition démocratique, soutenue avec enthousiasme sous le Front Populaire, et le régime de Vichy collaborant avec le fascisme et le nazisme.

5 Cette mémoire collective des minorités persécutées explique en particulier la forte proportion de femmes juives, les unes françaises ou naturalisées, issues du scoutisme, de formations politiques ou syndicales, d'autres, apatrides ou étrangères, souvent déjà actives avant-guerre dans des organisations spécifiques de gauche, sionistes ou communistes. Cette mémoire explique aussi la sensibilité particulière de la minorité protestante dont on connaît notamment l'admirable exemple du Chambon sur Lignon, village de 2500 habitants cachant et sauvant plusieurs milliers de juifs persécutés. Mentionnons aussi, à ce titre, le rôle de la CIMADE, en particulier de Madeleine Barot ${ }^{4}$, qui avait déjà aidé les réfugiés du IIIe Reich avant-guerre, de Laure Diebold, agent du réseau Mithridate, affectée en octobre 1942 au secrétariat de Jean Moulin, arrêtée et déportée à Ravensbrück, l'une des six femmes Compagnons de la Libération. Ou la doctoresse Adélaïde Hautval ${ }^{5}$, fille de pasteur, qui, pour avoir voulu empêcher l'arrestation de juifs, fut arrêtée et déportée à Auschwitz: Catherine Trautmann, maire de Strasbourg, vient d'attribuer son nom à une rue de la ville en remplacement de celui d'Alexis Carrel, certes prix Nobel de médecine, mais aussi défenseur d'un eugénisme mortifère, nommé par Pétain régent de la Fondation française pour l'étude des problèmes humains.

6 Sans être naturel, l'engagement des minoritaires pour la défense des Droits de l'homme et la liberté apparaît néanmoins comme une constante de l'Histoire. Majoritaires en France, les catholiques soutinrent d'abord largement l'État de Pétain jusqu'au sursaut de l'été 1942. Ce sursaut, dont une partie de la hiérarchie donna en quelque sorte le signal, fut provoqué surtout par le spectacle brutal des rafles de vieillards, de femmes et d'enfants juifs, qui revêt une importance particulière. Car, compte tenu de la tradition conservatrice et anti-démocratique de l'Église de France à cette époque, cet engagement ne relève pas seulement du patriotisme que la majorité catholique avait manifesté pendant la Première Guerre mondiale. Il témoigne d'un sens nouveau de la solidarité humaine qui, à travers en particulier les Amitiés chrétiennes ${ }^{6}$ - en fait, judéochrétiennes, bien qu'elles ne pussent s'appeler ainsi à l'époque -, va perdurer au-delà de la Résistance. C'est à Lyon que ce mouvement, qui allait après-guerre s'étendre des États-Unis à l'Europe occidentale, se manifesta d'abord, avec l'action conjuguée de responsables de l'œuvre juive de secours aux enfants (OSE) ${ }^{7}$, de résistants communistes, 
de catholiques - dont Germaine Ribière - et de protestants, pour arracher à la déportation les enfants juifs internés avec leurs parents au camp de Vénissieux et les cacher ensuite dans les institutions catholiques de la région avec la protection du cardinal Gerlier, pourtant intime de Pétain.

7 Comme le déclare Yvette Bernard-Fernoux ${ }^{8}$, qui remplaça Berthie Albrecht après l'arrestation de celle-ci à la tête du Service social du mouvement Combat, elle n'avait rien à perdre puisque, comme juive, elle était de toute façon condamnée à mort. Arrêtée à son tour, en janvier 1944, avec son mari, également membre de Combat, elle réussit à s'enfuir de l'hôpital où elle avait été tranférée pour accoucher d'un bébé mort sans doute des suites de l'arrestation. Pour des femmes socialistes militantes comme Suzanne Buisson, secrétaire du Comité national des femmes socialistes, déportée durant l'été 1943, ou communistes comme Danièle Casanova, fondatrice en 1940 de Comités féminins contre la guerre et le fascisme - ils aboutirent en 1943 à la création de l'Union des femmes françaises (UFF) - et déportée comme Marie-Claude VaillantCouturier à Auschwitz avec le convoi de Romainville, dit des 45 000, l'action s'inscrivait dans la logique de leur engagement politique d'avant-guerre. Ce qui était aussi le cas des syndicalistes.

8 Autre type d'engagement: celui de femmes qui, comme Germaine Tillion ou Louise Alcan', travaillant l'une au Musée de l'Homme, l'autre au Musée des Arts et Traditions populaires de Paris, mais on pourrait aussi citer le cas d'assistantes sociales, accédèrent à des réseaux de résistance par leurs relations professionnelles. Restent enfin celles qui rejoignirent la Résistance au nom d'une éthique personnelle, comme Marcelle Pardé, directrice du lycée de jeunes filles de Dijon, déportée avec sa secrétaire Simone Plessis à Ravensbrück où elles périrent, contribuant, avec plusieurs de mes enseignantes, à me sauver de la déportation. Pour certaines comme Dina Krischer, repliée et cachée à Lyon, ce fut simplement, dit-elle, le hasard si, sans avoir jamais fait partie d'un mouvement organisé, elle rejoignit le groupe Carmagnole de la FTP-MOI, où elle participa avec Simone Motta et Jacqueline Szynkmann aux mêmes sabotages, attentats et combats que les hommes. Elle dut même, alors qu'elle s'était réfugiée dans une cour près du Parc de la Tête d'Or lors d'une action en août 1944, abattre deux Allemands qui l'avaient découverte et allaient l'arrêter :

J'ai arraché leurs grades pour les donner à mon chef de groupe car quand on faisait une action seule, il fallait une preuve tangible. ${ }^{10}$

Certains exemples notoires comme celui de Lucie Aubrac qui organisa l'évasion de son mari, détenu par la Sipo SD de Lyon et fut l'une des rares femmes déléguées à l'Assemblée du Gouvernement provisoire d'Alger, celui de Danièle Casanova, figure emblématique de la résistance communiste ou de Marie-Madeleine Fourcade, responsable des 3000 agents du réseau Alliance rattaché à l'Intelligence Service, ne doivent pas faire oublier le traitement inégalitaire des résistantes par rapport à leurs compagnons de lutte. La diversité des tâches qui leur étaient confiées relevaient d'une spécificité le plus souvent assimilée à une complémentarité dévalorisante. Or, dans ses mémoires, Arsène Tchakarian, l'un des rares survivants du groupe ManouchianBoczow, reconnaît que leur travail était souvent beaucoup plus dangereux que celui de leurs compagnons combattant les armes à la main, «parce que si elles étaient prises, elles ne pouvaient pas se défendre $»^{11}$.

Rôle des femmes dans la Résistance 
11 Sans pouvoir détailler ici toutes les tâches qui leur étaient confiées, il est possible d'en esquisser une brève typologie. Celle d'agents de liaison impliquait en cas d'arrestation la déportation, voire l'exécution dans le cas de communistes comme Olga Bancic, guillotinée le jour de son trentième anniversaire à Stuttgart, France Bloch-Serrazin ou Simone Schloss transférées comme "Nuit et Brouillard» en Allemagne où elles subirent le même sort ; de même Renée Lévy, agrégée de lettres au lycée Victor Hugo, fille du grand rabbin de France, fut exécutée à Cologne. Autre tâche, le travail allemand (TA) fut confié à des jeunes femmes germanophones comme Jeannie Rousseau, interprète dans un organisme français travaillant avec les Allemands, membre du réseau Alliance. La situation est plus périlleuse encore pour des réfugiées allemandes ou autrichiennes, munies de faux papiers d'Alsaciennes, chargées d'infiltrer la Wehrmacht pour recueillir des renseignements et diffuser la propagande antinazie, voire envoyées pour organiser le sabotage dans des usines travaillant pour le IIIe Reich. Dans ce cas aussi, comme le raconte Tilly Spiegel ${ }^{12}$, l'arrestation impliquait la déportation à Ravensbrück ou la condamnation à mort par le «Tribunal du peuple » pour celles reconnues ressortissantes du Reich.

12 Jugées généralement incapables de se servir d'armes, les femmes, qui passaient plus facilement inaperçues avec un cabas ou une voiture d'enfants, effectuaient les repérages des lieux ainsi que les transports d'armes et d'explosifs avant les attentats ${ }^{13}$. Ariane Knout, dite Régine, du groupe de résistance La Forteresse juive, paya de sa vie cette action, alors qu'elle était secondée par sa fille Betty, à peine âgée de 16 ans. Annette Richtiger, 24 ans, remplaçant son mari Jean, FTP tué au combat, fut déchiquetée par une bombe alors qu'elle assurait le transport de la seule mitrailleuse disponible dans le secteur entre Lens et Valenciennes.

13 Dans la fabrication d'explosifs, hommes et femmes étaient le plus souvent associés. Ainsi, Boris et Hadassa Lerner, réfugiés de Bessarabie en France depuis 1938, fabriquaient des bombes et des explosifs en plein Quartier Latin, rue St. André des Arts. Arrêtés tous deux le 28 juin 1943, Boris fut fusillé, Hadassa survécut encore quelques années au retour de déportation. De même coopérèrent Taîbke Klescelski et le technicien Brumberger. Taîbke fut déchiquetée alors que tous deux transportaient pour vérification un nouvel explosif, de la banlieue parisienne dans un laboratoire de la capitale. Son compagnon n'est que légèrement touché. Ces exemples parmi d'autres montrent bien l'absurdité de la thèse de la complémentarité des rôles masculins et féminins, complémentarité au nom de laquelle Jeanne Bohec ${ }^{14}$, spécialiste des explosifs et, à ce titre, instructeure en sabotage dépendant du BCRA de la France Libre à Londres, se voit interdire, parce que femme, de participer aux combats de l'été 1944.

14 Toutes ces tâches, qualifiées généralement de "techniques ", auxquelles s'ajoute aussi la fabrication de faux papiers et cartes d'alimentation, de la presse et des tracts, tâches qui n'impliquaient pas moins, en cas d'arrestation, l'emprisonnement, voire la déportation, n'empêchaient pas les responsables masculins des organisations de résistance de demander à leurs « auxiliaires » féminines de les remplacer au pied levé lorsqu'ils étaient " grillés ». Ainsi, Adam Rayski est remplacé par Paulette RappaportGruda à la direction de l'Union des Juifs pour la Résistance et l'Entr'aide (UJRE) en zone Sud, Mina Puterflam est appelée à la direction FTP-MOI après les arrestations massives de l'été 1943, tandis que Thérèse Tennenbaum est nommée responsable dans les départements de l'Est et Catherine Varlin, déjà mentionnée, à un poste de commandement à Verdun dont aucun homme ne voulait et pour lequel elle se porte 
volontaire, afin de ne pas redevenir simple agent de liaison après avoir été l'une des responsables de la 35e Brigade FTP-MOI de Toulouse.

L'aspect le plus caractéristique de l'évaluation de la Résistance selon le schéma militaire classique de la lutte armée est l'occultation totale du rôle primordial des femmes dans le sauvetage des enfants juifs, alors que l'extermination des populations juives constituait en quelque sorte une guerre spécifique dans la guerre générale du Reich nazi. Certaines de ces femmes dont, notamment, Mila Racine, Nicole Salon-Weil, Suzanne Spaak, Marianne Cohn et bien d'autres encore, payèrent cette action de leur vie, mortes en déportation ou exécutées. Ainsi Suzanne Spaak, militante protestante, est fusillée en août 1944 à Fresnes, Marianne Cohn, alias Collin, réfugiée allemande, militante des Éclaireuses israélites de France, arrêtée lors d'un convoi clandestin d'enfants vers la Suisse, est arrachée de la prison d'Annemasse en juillet 1944, à 21 ans, violée et achevée par ses bourreaux à coups de bêche.

Dans cette guerre totale, qui n'épargnait ni les vieillards, ni les malades, ni les femmes et les enfants, les services alliés - comme le montre une recherche réalisée récemment par une de nos étudiantes de l'Université de Paris 7-Denis Diderot sur le retour des femmes juives déportées en France ${ }^{15}$ - avaient conçu le rapatriement selon le modèle de celui des prisonniers de la guerre 1914-1918. Ce qui explique le désarroi de ces femmes affaiblies, souvent malades, contraintes pour en finir avec une attente interminable de se débrouiller par leurs propres moyens pour atteindre un point de rapatriement, accueillies finalement avec si peu de compréhension que certaines, raconte Louise Alcan, préférèrent partir ensuite pour un temps à l'étranger ou se replier sur leurs proches et les Amicales de Déporté(e)s créées après la Libération.

Sous-estimation du rôle des femmes dans l'historiographie et la mémoire collective

Cette sous-estimation se reflète par voie de conséquence dans les manuels d'histoire. Comme si le courage civique qui devrait inspirer les jeunes d'aujourd'hui lorsqu'il s'agit de défendre dans la vie quotidienne ou dans des situations exceptionnelles une certaine idée de la liberté et des droits fondamentaux de toute personne humaine, ne pouvait s'identifier qu'à des hommes, alors que les survivantes se retrouvent encore aujourd'hui, par-delà les divergences d'opinion, pour défendre les valeurs pour lesquelles elles avaient combattu hier. Or, bien que le colonel Rol Tanguy ait affirmé dès la Libération «Dites bien que sans elles la moitié de notre travail aurait été impossible ${ }^{16}$, que les Françaises aient obtenu, en compensation, le droit de vote, reconnu aux femmes d'autres pays démocratiques bien avant elles, les résistantes ne représentent en moyenne que 2 à $3 \%$ des noms cités dans les ouvrages sur cette période. Et ce, jusqu'à la fin des annés 70. À partir de là, sans doute un effet de mai 68 et du nouveau féminisme, des rescapées de la déportation, notamment Charlotte Delbo, Anna Langfus, Germain Tillion, Brigitte Friang, commencent à rompre le silence. La première initiative d'envergure en ce sens est le Colloque de l'Union des femmes françaises organisé en 1975, à la Sorbonne, sur « les femmes dans la Résistance ${ }^{17}$, avec d'importants témoignages. Sur 17 ouvrages historiques abordant ce sujet, 13 sont postérieurs à 1971 ; les 4 autres paraissent entre 1948 et 1971. Même le grand colloque international du CNRS, en 1974, sur " la libération de la France ", publié deux ans plus tard, est muet sur le rôle des femmes et c'est encore à une journaliste que revient le mérite de poursuivre l'indispensable collecte de témoignages publiés en $1978^{18}$. L'essor contemporain de l'histoire orale aurait permis - on ne s'en aperçoit qu'actuellement - 
de procéder avec plus de rigueur méthodologique à l'enregistrement de témoins aujourd'huis disparus.

19 Mais il est un autre aspect de l'historiographie de la Résistance qui, tout en les concernant, dépasse la question des femmes et tient à une tradition fort ancienne de nationalisation de la culture par assimilation à la culture dominante. Cette tradition explique l'occultation du rôle des minorités, en particulier étrangères ou d'origine étrangère, surtout lorsque les intéressés n'ont pas eu le réflexe de franciser leur nom ou de garder leur identité de résistant. Et même dans ce cas, comme le souligne Catherine Varlin (Judith Heytin), le succès n'était pas garanti :

Quant au fait d'être femme, déclare-t-elle, je l'ai provisoirement oublié dès que m'ont été confiées des responsabilités de commandement... Les choses ne sont devenues plus complexes et plus gênantes que plus tard, après la guerre. Lorsque insidieusement d'abord, plus clairement ensuite, on a entrepris de purger la vie politique française de ses résistants, en particulier de ses résistants juifs. Lorsque la condition de femme a commencé à faire problème pour la reconnaissance des services rendus, pour la légitimation des grades, des décorations, etc. Il est vrai qu'être à la fois juive et femme n'a pas facilité l'entrée dans les manuels d'histoire officiels ou non. ${ }^{19}$

Encore ne mentionne-t-elle pas le handicap supplémentaire pour les étranger(e)s.

Comment s'étonner dans ces conditions qu'une aussi brillante journaliste ne se soit résolue à aborder le problème qu'à l'occasion du Colloque pour la recherche sur l'histoire contemporaine des Juifs (RHICOJ) organisé au Sénat en 1984 et que la minorité juive, notamment ceux et celles qui avaient le plus souffert de leur exclusion, ait été la première dans les années 1970 à s'insurger contre l'occultation de son histoire ${ }^{20}$. En ce sens, David Diamant fait œuvre de pionnier en ajoutant au titre de son ouvrage déjà mentionné sur la résistance des juifs le sous-titre «Avec ou sans armes ». Ce qui lui permet de mentionner 100 femmes sur les 600 noms cités de résistants, soit $16,7 \%$ et 110 noms de femmes, soit $14 \%$, dans le martyrologe publié en annexe. Dans $L a$ Résistance organisée des Juifs de France (Julliard, 1973), Jacques Ravine relève pour sa part que sur les 69 membres de la résistance juive arrêtés lors des descentes particulièrement meurtrières de l'été 1943, 41 étaient des femmes. Proportion importante aussi, soit $20 \%$ de résistantes, citée par Alain Michel dans son ouvrage sur Les éclaireurs israélites de France pendant la Deuxième Guerre mondiale (Paris, éd. des EIF, 1984). Si ces statistiques partielles qui exigeraient un recensement plus complet ont le mérite de donner une idée de l'importance de l'engagement des femmes, celui-ci reste cependant minimisé, même lorsqu'il est attesté par bon nombre d'historiens. Ainsi peut-on lire dans le texte d'un universitaire grenoblois sur le groupe CarmagnoleLiberté des FTP-MOI : "Peut-être convient-il de dire quelques mots aussi de la participation des femmes à la lutte des FTP-MOI", tout en soulignant par ailleurs qu'elles constituaient $25 \%$ de ce groupe ${ }^{21}$. C'est grâce au témoignage récent d'un survivant de la 35e Brigade FTP-MOI de Toulouse ${ }^{22}$, que nous connaissons désormais au moins les noms de douze résistantes de ce groupe - dont Rosine Bet, déjà mentionnée par ailleurs, tuée lors de l'attentat contre le cinéma de propagande allemande «Variétés »- et de cinq déportées.

22 Mais c'est essentiellement jusqu'à présent grâce à Denise Lorach, rescapée de BergenBelsen, conservatrice du Musée de la Résistance et de la Déportation de Besançon, et, plus récemment, à Sabine Zeitoun, qui a soutenu à l'Université Paris 7-Denis Diderot une thèse sur l'OSE et le sauvetage des enfants juifs et dirige maintenant le Centre 
d'Histoire de la Résistance et de la Déportation à Lyon, grâce aussi à quelques réalisatrices de documentaires, que cette "ville engloutie» qu'évoque Catherine Varlin, émerge peu à peu. Dans sa contribution, déjà mentionnée, sur «Les Françaises sous Vichy ", Hélène Eck, l'une des rares historiennes à évoquer la question, souligne que « l'impôt du sang n'a pas suffi à fonder l'égalité ». Du moins peut-on espérer que les historiennes des nouvelles générations auront à cœur de rendre un nom, un visage, à celles qui se sont engagées au péril de leur vie pour défendre la liberté et la démocratie dont elles bénéficient aujourd'hui.

\section{NOTES}

1. Catherine Varlin, « Une ville engloutie : la résistance des femmes juives », in RHICOJ, Les Juifs dans la Résistance et la Libération, Paris, éd. du Scribe, 1985, pp. 101-103.

2. Colloques du 7 octobre 1984 au Sénit, publié par RHICOJ, op. cit., pp. 89-94, et du 22 juin 1994 à l'Assemblée nationale, in Le Monde Juif, Paris, CDJC, septembre-octobre 1994, pp. 177-182.

3. Cf. notamment: Jean-Charles Bonnet, Les pouvoirs publics et l'immigration dans l'entredeux-guerres, thèse de troisième cycle, Universit de Lyon II, Centre Pierre Léon, 1974 ; Ralph Schor, L'immigration en France 1919-1939. Sources imprimées en langue française et filmographie, Université de Nice, 1986 ; Gérard Noiriel, Population immigrée et identité nationale en France XIXe-XXe siècle, Hachette, 1992 ; Rita Thalmann, « Les réfugiés du IIIe Reich : un cas particulier de l'immigration dans la France des années trente ", in Combat pour la Diaspora, Paris, 1986, n 18-19, pp. 37-46.

4. Madeleine Barot, « La CIMADE et les camps d'internement de la zone Sud 1940-1944 », in Xavier de Montclos et al., Églises et chrétiens dans la Deuxième Guerre mondiale (La France), actes du colloque de Lyon 1978, Lyon, PUL, 1982.

5. Dr. Adélaïde Hautval, Médecine et crimes contre l'humanité, éd. Actes Sud, 1991.

6. Renée Bédarida, Les armes de l'esprit. Témoignage chrétien 1941-1944, Paris, éd. Ouvrières, 1977.

7. Sabine Zeitoun, L'œuvre de secours aux enfants (OSE) sous l'Occupation en France, Paris, L'Harmattan, 1990, et Ces enfants qu'il fallait sauver, Albin Michel, 1990.

8. Yvette Bernard-Fernoux, "A la suite de Berthie Albrecht au Service social des MUR », in RHICOJ, op. cit., pp. 104-108.

9. Germaine Tillion, Ravensbrück, 1e éd. 1946, 2e et 3e éd. augm. Le Seuil, 1972 et 1988 ; Louise Alcan, Le temps écartelé, St. Jean de Maurienne, 1980.

10. Dina Krischer, «Combattante à Carmagnole », in RHICOJ, op. cit., pp. 98-100.

11. Cité in Marie-Louise Coudert, elles la résistance, Paris, Messidor, 1983, p. 163.

12. Tilly Spiegel, Frauen und Mädchen im österreichischen Widerstand, Wien-FrankfurtZürich, 1966. Lors de notre rencontre à Vienne, Tilly Spiegel m'a raconté qu'une de ses amies et compagnes de lutte, qui attendait un enfant lors de son arrestation, a préféré se jeter par la fenêtre et mourir par crainte de livrer des camarades. 
13. David Diamant, Les Juifs dans la Résistance française (1940-1944) avec et sans armes, Paris, Le Pavillon, 1971 ; Rita Thalmann, « Une lacune de l'historiographie », in RHICOJ, op. cit., pp. 89-94.

14. Hélène Eck, «Les Françaises sous Vichy », in Georges Duby et Michelle Perrot, Histoire des femmes, t. 5, Le XXe siècle (sous la direction de Françoise Thébaud), Plon, 1992, pp. 185-211.

15. Sylvie Lalario, Retour en France et réadaptation des femmes juives déportées, Mémoire de maîtrise d'histoire, Université Paris 7-Denis Diderot, 1993.

16. Yvonne Féron, La Libération de Paris, Hachette, 1945.

17. Actes du Colloque de l'Union des femmes françaises de novembre 1975, Paris, éd. du Rocher, 1977.

18. Ania Francos, Il était des femmes dans la Résistance, Paris, Stock, 1978.

19. Catherine Varlin, « Une ville engloutie : les femmes juives dans la Résistance », in RHICOJ, op. cit., p. 102.

20. Annette Wieviorka, Ils étaient juifs, résistants, communistes, éd. Denoël, 1985 ; Déportation et génocide. Entre la Mémoire et l'Oubli, Plon, 1992.

21. Claude Collin, "Carmagnole et Liberté : les FTP-MOI à Lyon et Grenoble », in Philippe Joutard-François Marcot (éd.), Les étrangers dans la Résistance en France, catalogue de l'exposition du Musée de la Résistance et de la Déportation, Besançon, octobre 1992, p. 121, aussi in Le monde juif, op. cit., p. 117.

22. Marc Brafman, témoignage sur la 35e Brigade FTP-MOI (Toulouse), in Le monde juif, op. cit., pp. 79-95.

\section{RÉSUMÉS}

Bien que le colonel Rol-Tanguy ait déclaré dès la Libération que «sans elles, la moitié de notre travail eût été impossible», qu'elles aient obtenu en 1944 le droit de vote, reconnu depuis longtemps aux femmes d'autres pays démocratiques, les résistantes restent les grandes oubliées de l'historiographie des années 1940-1944. Pourtant, par leur origine et leur statut dans la société française de l'époque, leur engagement et les missions qui leur étaient confiées ne comportaient pas moins de risques que ceux de leurs compagnons de lutte. Cette occultation persistante tient essentiellement à deux facteurs: 1) la dévalorisation de leur rôle jugé secondaire, complémentaire par rapport au service armé des hommes. 2) la nationalisation à posteriori de la Résistance, conduisant à passer sous silence l'importante contribution à la libération de la France des étrangers, des étrangères ou des personnes d'origine étrangère.

Rita Thalmann : The Forgetting of Women in Resistance Historiography

Women in the Resistance are largely absent in the historiography of the years 1940-1944, despite the fact that Colonel Rol-Tanguy declared at the Liberation that "without (women), half our work could not have be done ", and despite that women got the vote in 1944 (years after women in other democratic countries). Given their origins and their status in French society, however, the missions women undertook and their commitment to the cause were no less risky than those undertaken by their male companions. The persistent silence concerning women in the Resistance can be traced to two main causes : 1) the devalorisation of theirs roles, which are 
relegated to a secondary and complementary position next to men's armed services 2) the way the Resistance was "nationalized» after the fact has contributed to silencing the important contributions of foreigners or of foreign born persons in the liberation of France

\section{AUTEUR}

\section{RITA THALMANN}

Rita THALMANN est professeur émérite à l'Université Paris 7-Denis Diderot, spécialiste du monde germanique. Ses principaux ouvrages sont : La nuit de cristal (1972), Protestantisme et nationalisme en Allemagne de 1900 à 1945 (sa thèse publiée en 1976), Être femme sous le IIIe Reich (1982), La mise au pas de la France (1992). Auteure de nombreux articles sur les juifs, les émigrés du IIIe Reich et les femmes, elle a aussi dirigé deux ouvrages collectifs : Femmes et fascismes (1986) et La tentation nationaliste (1990). 